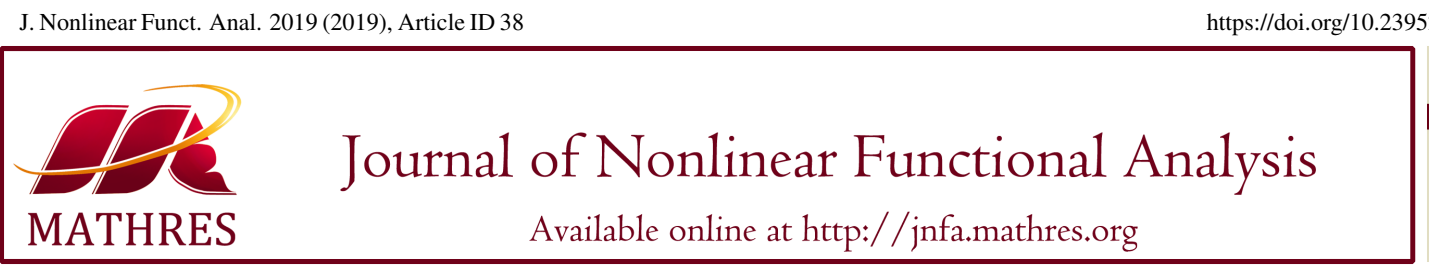

\title{
A PRIMAL-DUAL INTERIOR-POINT METHOD FOR LINEAR OPTIMIZATION BASED ON A NEW PARAMETERIZED KERNEL FUNCTION
}

\author{
MENGMENG LI ${ }^{1}$, MINGWANG ZHANG ${ }^{1, *}$, ZHENGWEI HUANG $^{2}$ \\ ${ }^{1}$ Three Gorges Mathematical Research Center, China Three Gorges University, Yichang 443002, China \\ ${ }^{2}$ College of Economics and Management, China Three Gorges University, Yichang 443002, China
}

\begin{abstract}
As recently demonstrated by the study of the primal-dual interior-point methods based on kernel functions, a kernel function not only serves to determine the search direction and measure the distance of the current iteration point to the $\mu$-center, but also affects the iteration complexity and the practical computational efficiency of the algorithm. In this paper, we propose a primal-dual interior-point method for a linear optimization based on a new parameterized kernel function. The construction of the new parameterized kernel function is motivated by the parameterized ways of existing kernel functions. By using properties of the new parameterized kernel function, we improve the iteration bound of the large-update method from $O\left(n^{\frac{3}{4}} \log \frac{n}{\varepsilon}\right)$ to $O\left(\sqrt{n} \log n \log \frac{n}{\varepsilon}\right)$, which is the best theoretical iteration result currently known. Finally, some numerical results are given to present the efficiency and potential of our kernel function.
\end{abstract}

Keywords. Large-update method; Linear optimization; Primal-dual interior-point method; Parameterized kernel function.

2010 Mathematics Subject Classification. 90C51, 90C05.

\section{INTRODUCTION}

Since the groundbreaking paper of Karmarkar in 1984 [1], many scholars have been committed to the study of primal-dual interior-point methods (IPMs) for linear optimization (LO) and numerous results have been obtained; see, e.g., [2, 3, 4, 5]. Among the multifarious variants of IPMs, the primal-dual IPM is generally believed as the most efficient one in terms of computational efficiency [6]. A basic method of primal-dual IPMs is to approximate the optimal solution along the central path. The definition of the central path for primal-dual IPMs was initially described by Megiddo in 1989 [7], and the evolution of primal-dual IPMs was surveyed in Kojima, Mizuno and Yoshise [5] and Gonzaga [8].

In 1992, Roos and Vial [9] pointed out that the essence of the Karmarkar's IPM was to solve an optimization problem related to the classical logarithmic function. In 1994, Nesterov and Nemirovskii [10] presented an elegant and simple barrier function, motivating the run of the algorithm in polynomial

\footnotetext{
${ }^{*}$ Corresponding author.

E-mail addresses: mengmengli07@163.com (M. Li), zmwang@ctgu.edu.cn (M. Zhang), zhengweihuang@ctgu.edu.cn (Z. Huang).

Received January 22, 2019; Accepted August 8, 2019.
}

(C) 2019 Journal of Nonlinear Functional Analysis 
time which is called as the self-concordant barrier function. Recently, Peng, Roos and Terlaky [11] made an important contribution to the IPMs based on kernel functions. They introduced a special kind of kernel function called self-regular kernel function whose prototype is given by

$$
\Upsilon_{p, q}(t)=\frac{t^{p+1}-1}{p(p+1)}+\frac{t^{1-q}-1}{q(q-1)}+\frac{p-q}{p q}(t-1), t>0, p \geq 1, q>1 .
$$

The complexity bound of the large-update primal-dual IPM for linear optimization was significantly improved from $O\left(n \log \frac{n}{\varepsilon}\right)$ to $O\left(\sqrt{n} \log n \log \frac{n}{\varepsilon}\right)$ based on the self-regular kernel function [12].

Furthermore, Bai, Ghami and Roos [13] relaxed the restrictions of the self-regular kernel function and presented a series of eligible kernel functions, which obtained the same iteration bounds as Peng et al. in [11]. The kernel function with trigonometric barrier was first analyzed by Ghami et al. [14]. Recently, a lot of (trigonometric) kernel functions have been proposed. Moreover, the methods of solving LO based on a kernel function have been extended to various optimization problems, for instance, semidefinite optimization (SDO), $P_{*}(\kappa)$ linear complementarity problem (LCP), and second order cone optimization (SOCO). Specifically, Li and Zhang [15] proposed a new trigonometric kernel function, and developed some new analytic tools to analyze the complexity of the algorithm. Ji, Zhang and $\mathrm{Li} \mathrm{[16]} \mathrm{proposed}$ a large-update primal-dual IPM for $P_{*}(\kappa)$ LCPs based a new class kernel function, which is neither classical logarithmic nor self-regular function.

Recently, Fathihafshejani, Jahromi and Peyghami [17] presented a new generic trigonometric kernel function, and gave the iteration bound and numerical results of the algorithm for SDO. For more studies with primal-dual IPMs based on a kernel function, we refer to [18, 19, 20, 21, 22].

In particular, Bai, Ghami and Roos [23] presented the following parameterized kernel function with a finite barrier

$$
\psi_{b 1}(t)=\frac{t^{2}-1}{2}+\frac{1}{b}\left(e^{b(1-t)}-1\right), t>0, b>0,
$$

where the parameter $b$ not only represents a barrier degree, but also plays a important role in improving the iteration complexity of the algorithm. To the best of our knowledge, this is the first work in which a barrier parameter on a kernel function is considered in the context. Thereafter, parameterized kernel functions are proposed one after another, most of which are generalizations of the existing kernel functions, and the iteration bounds of the large-update or small-update methods based on these parameterized kernel functions for LO are improved. Table 1 gives several known kernel functions which improve the iteration bounds of the primal-dual large-update methods for LO by adding parameters to the existing kernel functions.

Motivated by the previous research, in this paper we introduce a parameterized version of a kernel function that was previously presented by Bai, Roos, and Ghami [24] in 2002. Although almost all the early proposed kernel functions have been parameterized, to our knowledge, its parameterized version has not been put forward yet. To construct a new parameterized kernel function, we first investigate several one defined in literature and derive some conditions and then by applying these conditions and some parameterized ways of existing kernel functions, a new parameterized kernel function is defined and its properties are introduced. The new parameterized kernel function is not self-regular, but it is eligible. By these good properties, the complexity of the large-update algorithm for LO is reduced from $O\left(n^{\frac{3}{4}} \log \frac{n}{\varepsilon}\right)$ to $O\left(\sqrt{n} \log n \log \frac{n}{\varepsilon}\right)$, which is consistent with the best known results. We also give few numerical results to verify the efficiency of our kernel function. 


\begin{tabular}{|c|c|c|c|}
\hline Kernel function & Parameterized kernel function & Iteration bounds & References \\
\hline \multirow{2}{*}{$\frac{t^{2}-1}{2}+\frac{1-t^{2}}{2 t^{2}}$} & $\frac{t^{2}-1}{2}+\frac{t^{1-q}-1}{q-1}, q>1$ & $O\left(n^{\frac{2}{3}} \log \frac{n}{\varepsilon}\right) \rightarrow O\left(\sqrt{n} \log n \log \frac{n}{\varepsilon}\right)$ & \multirow{2}{*}[12,13,25]{} \\
\hline & $\frac{t^{2}-1}{2}+\frac{t^{1-q}-1}{q(q-1)}-\frac{q-1}{q}(t-1), q>1$ & $O\left(n^{\frac{2}{3}} \log \frac{n}{\varepsilon}\right) \rightarrow O\left(\sqrt{n} \log n \log \frac{n}{\varepsilon}\right)$ & \\
\hline \multirow{2}{*}{$\frac{t^{2}-1}{2}+e^{\frac{1}{t}-1}-1$} & $\frac{t^{2}-1}{2}+\frac{q^{\frac{1}{t}-1}-1}{\log q}, q>1$ & $O\left(\sqrt{n} \log ^{2} n \log \frac{n}{\varepsilon}\right) \rightarrow O\left(\sqrt{n} \log n \log \frac{n}{\varepsilon}\right)$ & \multirow{2}{*}[13,26,27]{} \\
\hline & $\frac{t^{2}-1}{2}+\frac{1}{p q}\left(e^{p\left(e^{q\left(\frac{1}{t}-1\right)}-1\right)}-1\right), p, q \geq 1$ & $O\left(\sqrt{n} \log ^{2} n \log \frac{n}{\varepsilon}\right) \rightarrow O\left(\sqrt{n} \log n \log \frac{n}{\varepsilon}\right)$ & \\
\hline$\frac{t^{2}-1}{2}-\int_{1}^{t} e^{\frac{1}{\xi}-1} d \xi$ & $\frac{t^{2}-1}{2}-\int_{1}^{t} e^{q\left(\frac{1}{\xi}-1\right)} d \xi, q \geq 1$ & $O\left(\sqrt{n} \log ^{2} n \log \frac{n}{\varepsilon}\right) \rightarrow O\left(\sqrt{n} \log n \log \frac{n}{\varepsilon}\right)$ & {$[13,28]$} \\
\hline \multirow[t]{2}{*}{$\frac{t^{2}-1}{2}+\left(\frac{1}{t}-1\right) e^{\frac{1}{t}-1}$} & $\frac{t^{2}-1}{2}+\frac{1}{q}\left(\frac{1}{t^{q}}-1\right) e^{\frac{1}{t^{q}}-1}, q>1$ & $\begin{array}{c}O\left(\sqrt{n} \log ^{2} n \log \frac{n}{\varepsilon}\right) \rightarrow \\
O\left(\sqrt{n}[\log (\log n)](\log n)^{\left.1+\frac{1}{\log (\log n)} \log \frac{n}{\varepsilon}\right)}\right.\end{array}$ & \multirow[t]{2}{*}{ [29] } \\
\hline & $\frac{t^{2}-1}{2}+\frac{1}{q^{2}}\left(\frac{q}{t}-1\right) e^{q\left(\frac{1}{t}-1\right)}-\frac{q-1}{q^{2}}, q \geq 1$ & $O\left(\sqrt{n} \log ^{2} n \log \frac{n}{\varepsilon}\right) \rightarrow O\left(\sqrt{n} \log n \log \frac{n}{\varepsilon}\right)$ & \\
\hline
\end{tabular}

TABLE 1. Several known kernel functions

The structure of this paper is given below: In Section 2, we recall the notions of the central path and search direction, which are the basic concepts of the primal-dual IPMs for the LO. We also provide a generic primal-dual IPM for the LO. Section 3 is devoted to constructing a new parameterized kernel function, whose properties are introduced in Section 4. The convergence analysis of the method is investigated in Section 5. Moreover, some numerical results are presented in Section 6. Finally, some concluding remarks are drawn in Section 7.

Some notations used throughout the paper are as follows. $R^{n}$ denotes the set of $n$-dimensional vectors. the set of $n$-dimensional nonnegative vectors and positive vectors are defined as $R_{+}^{n}$ and $R_{++}^{n}$, respectively. Furthermore, $e$ means $n$-dimensional vector of ones. $\|\cdot\|$ denotes the 2 -norm of a vector. The coordinatewise product of the vectors $x$ and $s$ is represented as $x s$ for any $x, s \in R^{n}$, and $x_{i}$ denotes the $i$ th component of vector $x$ with $1 \leq i \leq n$. Finally, if $f: R_{+} \rightarrow R_{+}$and $x \in R_{+}^{n}$, then $f(x)$ is used to indicate a vector in $R_{+}^{n}$ whose $i$ th component is $f\left(x_{i}\right)$. The formula $f(x)=O(g(x))$ denotes $f(x) \leq c g(x)$ for some positive constant $c$, and $f(x)=\Theta(g(x))$ denotes $c_{1} g(x) \leq f(x) \leq c_{2} g(x)$ for positive constants $c_{1}$ and $c_{2}$.

\section{PRELIMINARIES}

2.1. The central path. Consider the standard form of the LO

$$
\text { (P) } \min \left\{c^{T} x: A x=b, x \geq 0\right\}
$$

and its dual problem

(D) $\max \left\{b^{T} y: A^{T} y+s=c, s \geq 0\right\}$, 
where $A \in R^{m \times n}, \operatorname{rank}(A)=m, b, y \in R^{m}$ and $c, x, s \in R^{n}$. It is known that the optimal solution to the (P) and the $(\mathrm{D})$ is equivalent to the solution to the following system:

$$
\begin{cases}A x=b, & x \geq 0, \\ A^{T} y+s=c, & s \geq 0, \\ x s=0 . & \end{cases}
$$

The third equation $x s=0$ in (2.1) is so-called complementarity condition for the (P) and the (D), and the basic idea of primal-dual IPMs is to replace the complementarity condition $x s=0$ in (2.1) by the parameterized equation $x s=\mu e(\mu>0)$. Therefore, we consider the system as below:

$$
\begin{cases}A x=b, & x \geq 0, \\ A^{T} y+s=c, & s \geq 0, \\ x s=\mu e . & \end{cases}
$$

A solution of (2.2) exists if and only if (P) and (D) satisfy the interior-point condition (IPC) [2], i.e., there exists $\left(x^{0}, s^{0}, y^{0}\right)$ such that

$$
A x^{0}=b, x^{0}>0, A^{T} y^{0}+s^{0}=c, s^{0}>0 .
$$

Without loss of generality, we assume that the IPC is satisfied. Then, for each $\mu>0$, a unique solution of (2.2) exists [2]. The solution of (2.2) is denoted as $(x(\mu), s(\mu), y(\mu))$, where $x(\mu)$ is called the $\mu$-center of (P) and $(s(\mu), y(\mu))$ is called the $\mu$-center of (D). The set of $\mu$-center gives a homotopy path, which is called the central path of (P) and (D) [30]. If $\mu \rightarrow 0$, then the limit of the central path yields optimal solutions for (P) and (D) [2].

2.2. The search direction. The search direction of the primal-dual IPMs is determined by Newton's method, and this yields the following equations:

$$
\left\{\begin{array}{l}
A \Delta x=0, \\
A^{T} \Delta y+\Delta s=0, \\
x \Delta s+s \Delta x=\mu e-x s .
\end{array}\right.
$$

Define

$$
d_{x}:=\frac{v \Delta x}{x}, d_{s}:=\frac{v \Delta s}{s}, \text { where } v:=\sqrt{\frac{x s}{\mu}} .
$$

It is easy to see that (2.4) can be rewritten the following system:

$$
\left\{\begin{array}{l}
\bar{A} d_{x}=0, \\
\bar{A}^{T} \Delta y+d_{s}=0, \\
d_{x}+d_{s}=v^{-1}-v,
\end{array}\right.
$$

where

$$
\bar{A}:=\frac{1}{\mu} A V^{-1} X, V:=\operatorname{diag}(v), X:=\operatorname{diag}(x) .
$$

The third equation in (2.6) is called the scaled centering equation, and it is exactly the negative gradient of the classical logarithmic barrier function, which is given by

$$
\Psi_{c}(v):=\sum_{i=1}^{n}\left(\frac{v_{i}^{2}-1}{2}-\log v_{i}\right)
$$


In this paper, we replace the classical logarithmic barrier function $\Psi_{c}(v)$ by a new barrier function $\Psi(v)$. The barrier function $\Psi(v)$ is determined by the univariate kernel function $\psi(t)$. A twice differentiable function $\psi(t): R_{++} \rightarrow R_{+}$is called a kernel function if $\psi(t)$ satisfies the following conditions:

$$
\psi(1)=\psi^{\prime}(1)=0 ; \psi^{\prime \prime}(t)>0, \forall t>0 ; \lim _{t \downarrow 0} \psi(t)=\lim _{t \rightarrow \infty} \psi(t)=+\infty .
$$

Obviously, the kernel function $\psi(t)$ attains its minimal value at $t=1$ and goes to infinity if either $t \downarrow 0$ or $t \rightarrow \infty$. Hence $\psi(t)$ can be completely determined by its second derivative as follows:

$$
\psi(t)=\int_{1}^{t} \int_{1}^{\xi} \psi^{\prime \prime}(\zeta) d \zeta d \xi
$$

The barrier function $\Psi(v)$ determined by its kernel function $\psi(t)$ is

$$
\Psi(v):=\sum_{i=1}^{n} \psi\left(v_{i}\right)
$$

It is apparent that the kernel function of the classical logarithmic barrier function is

$$
\psi_{c}(t)=\frac{t^{2}-1}{2}-\log t .
$$

Replaced $\Psi_{c}(v)$ by the new barrier function $\Psi(v)$, system (2.6) can be rewritten as

$$
\left\{\begin{array}{l}
\bar{A} d_{x}=0, \\
\bar{A}^{T} \Delta y+d_{s}=0, \\
d_{x}+d_{s}=-\nabla \Psi(v) .
\end{array}\right.
$$

Now one easily obtains the scale search direction $d_{x}$ and $d_{s}$ by solving (2.10), and we can derive the centering search direction $(\Delta x, \Delta y, \Delta s)$ by (2.5). Note that the vector $d_{x}$ belongs to the null space of the matrix $\bar{A}$ and $d_{s}$ belongs to the row space of the matrix $\bar{A}$. Thus $d_{x}$ and $d_{s}$ are orthogonal vectors, i.e., $d_{x}^{T} d_{s}=0$. Hence, $d_{x}+d_{s}=0 \Leftrightarrow \nabla \Psi(v)=0 \Leftrightarrow v=e \Leftrightarrow \Psi(v)=0$. From what has been discussed above, we could draw the conclusion that if $(x, y, s) \neq(x(\mu), y(\mu), s(\mu))$, then $(\Delta x, \Delta y, \Delta s) \neq 0$.

A generic primal-dual algorithm for LO is given in Figure 1 as below:

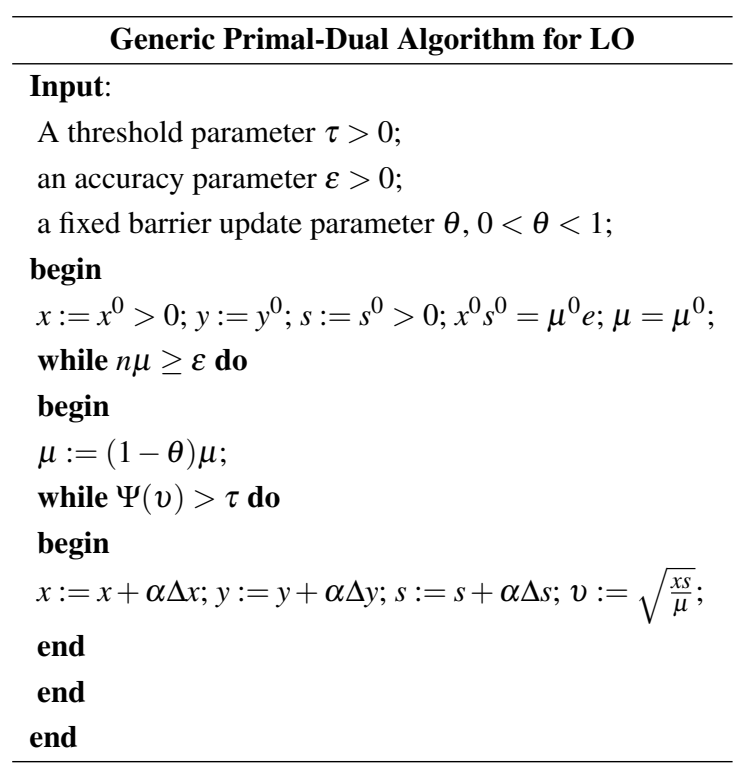


The threshold parameter $\tau$, accuracy parameter $\theta$ and the step-size $\alpha$ described in the algorithm are chosen to ensure that the number of iterations is as small as possible. Moreover, if the parameter $\theta$ is a constant and is independent of the dimension $n$ of the problem, such as $\theta=\frac{1}{2}$, then the algorithm is called a large-update method. If the parameter $\theta$ is dependent on the dimension $n$ of the problem, for instance, $\theta=\frac{1}{\sqrt{n}}$, then we call the algorithm a small-update method.

In the theoretical analysis, the small-update methods are much more efficient than the large-update methods, however, in practice, large-update methods perform better [2, 3, 4]. This implies that there is a gap between the theoretical behavior and practical computational efficiency of the algorithm [31]. In this paper, we mainly analyze large-update methods.

\section{THE NEW KERNEL FUNCTION}

This section is devoted to constructing a new parameterized kernel function that improves the iteration complexity of the algorithm by adding a suitable parameter to an existing kernel function. We first study some kernel functions and its parameterized versions defined in literature. Motivated by the parameterized ways of these kernel functions, a new parameterized kernel function is defined.

The original kernel function introduced by Bai, Roos and Ghami in [24] is given as follows:

$$
\psi_{A}(t)=\frac{t^{2}-1}{2}+\frac{(e-1)^{2}}{e\left(e^{t}-1\right)}-\frac{e-1}{e}, t>0,
$$

and the complexity bound of the large-update method based on this kernel function is $O\left(n^{\frac{3}{4}} \log \frac{n}{\varepsilon}\right)$. Finding in (3.1) and any kernel function in the Table 1 can be rewritten as follows:

$$
\psi(t)=\frac{t^{2}-1}{2}+\psi_{b}(t)
$$

where function $\frac{t^{2}-1}{2}$ is named growth term and $\psi_{b}(t)$ is called barrier term. It is clear that (3.1) and all kernel functions in Table 1 satisfy the following conditions:

Condition 3.1. For (3.1) and all kernel functions defined in Table 1, the barrier terms satisfy

- $\psi_{b}(t)$ is convex and strictly decreasing function for all $t>0$;

- $\psi_{b}(1)=0 ; \psi_{b}^{\prime}(1)=1 ; \lim _{t \downarrow 0} \psi_{b}(t)=+\infty ; \lim _{t \rightarrow \infty} \psi_{b}(t)=$ constant .

Condition 3.2. For (3.1) and all kernel functions defined in Table 1, we have

- $\psi^{\prime \prime}(t)>1$;

- $\psi^{\prime}(t)+t \psi^{\prime \prime}(t) \geq 0$;

- $\psi^{\prime \prime \prime}(t)<0$.

In fact, a twice differentiable function is called exponential convex or e-convex if it satisfies the property as below:

$$
\psi^{\prime}(t)+t \psi^{\prime \prime}(t) \geq 0
$$

This property has been proved to be essential in analyzing the convergence of the primal-dual IPMs based on kernel functions. In the following lemma we list some formulas that are equivalent to (3.3).

Lemma 3.3. ([11] Lemma 2.1.2) The following three formulas are equivalent if $\psi(t)$ is a twice differentiable function for $t>0$ : 
(i) $\psi\left(\sqrt{t_{1} t_{2}}\right) \leq \frac{1}{2}\left(\psi\left(t_{1}\right)+\psi\left(t_{2}\right)\right), t_{1}, t_{2}>0 ;$

(ii) $\psi^{\prime}(t)+t \psi^{\prime \prime}(t) \geq 0, t>0$;

(iii) $\psi\left(e^{\xi}\right)$ is convex.

Table 1 provides several known kernel functions which improve the iteration bounds of the primaldual large-update methods for LO by adding parameters to the existing kernel functions. From the above discussion, we conclude that (3.1) and all kernel functions defined in Table 1 satisfy some common conditions. Thus, by using these features, a new parameterized kernel function corresponding to (3.1) can be defined. Furthermore, what we want is that the new parameterized kernel function can improve the iteration complexity of the method. It is natural to ask how to add a proper value of the parameter for a better complexity bound.

By studying the parameterized ways of these kernel functions and others in the literature, several common types of barrier terms and its corresponding parameterized versions are as follows:

- $\frac{1}{t}-1 \Longrightarrow \frac{1}{t^{q}}-1$

- $e^{\frac{1}{t}-1}-1 \Longrightarrow(1) e^{q\left(\frac{1}{t}-1\right)}-1 ;(2) q^{\frac{1}{t}-1}-1$

Inspired by the parameterized ways of the above two barrier terms and (3.1), we construct a barrier term

$$
\psi_{b}(t)=\frac{(e-1)^{q+1}}{q e\left(e^{t}-1\right)^{q}}-\frac{e-1}{q e}, t>0, q \geq 1 .
$$

Then we get the corresponding parameterized kernel function

$$
\psi(t)=\frac{t^{2}-1}{2}+\frac{(e-1)^{q+1}}{q e\left(e^{t}-1\right)^{q}}-\frac{e-1}{q e}, t>0, q \geq 1 .
$$

It is easy to verify that the new parameterized kernel function satisfies Condition 3.1 and Condition 3.2. We will prove that the iteration bound of the large-update algorithm is improved.

\section{SOME PROPERTIES OF THE NEW KERNEL FUNCTION}

This section aims to investigate some properties of the new kernel function. In the following convergence analysis of the method, we use the norm-based proximity measure $\delta(v)$ defined by

$$
\delta(v):=\frac{1}{2}\|\nabla \Psi(v)\|=\frac{1}{2}\left\|d_{x}+d_{s}\right\| .
$$

It is known from the definition of the barrier function that $\Psi(v)$ is strictly convex and the minimum value is obtained at $v=e$. Thus

$$
\Psi(v)=0 \Leftrightarrow \delta(v)=0 \Leftrightarrow v=e .
$$

Bai, Ghami and Roos [13] gave five more conditions on the kernel function, that is,

$$
\begin{aligned}
& t \psi^{\prime \prime}(t)+\psi^{\prime}(t)>0, t<1 \\
& t \psi^{\prime \prime}(t)-\psi^{\prime}(t)>0, t>1 \\
& \psi^{\prime \prime \prime}(t)<0, t>0 \\
& 2 \psi^{\prime \prime}(t)^{2}-\psi^{\prime}(t) \psi^{\prime \prime \prime}(t)>0, t<1 \\
& \psi^{\prime \prime}(t) \psi^{\prime}(\beta t)-\beta \psi^{\prime}(t) \psi^{\prime \prime}(\beta t)>0, t>1, \beta>1 .
\end{aligned}
$$


For simplicity of presentation, let $M=\frac{(e-1)^{q+1}}{e}$. Some straightforward calculations yield the first three derivatives of the kernel function $\psi(t)$ as follows:

$$
\begin{aligned}
& \psi^{\prime}(t)=t-\frac{M e^{t}}{\left(e^{t}-1\right)^{q+1}}, \\
& \psi^{\prime \prime}(t)=1+\frac{M\left(q e^{2 t}+e^{t}\right)}{\left(e^{t}-1\right)^{q+2}} \\
& \psi^{\prime \prime \prime}(t)=-\frac{M\left[q^{2} e^{3 t}+(3 q+1) e^{2 t}+e^{t}\right]}{\left(e^{t}-1\right)^{q+3}} .
\end{aligned}
$$

Following [13], a kernel function $\psi(t)$ is called an eligible kernel function if $\psi(t)$ satisfies conditions (4.2a), (4.2c), (4.2d) and (4.2e). Moreover, If $\psi(t)$ satisfies (4.2b) and (4.2c), then $\psi(t)$ satisfies (4.2e). Now one easily checks that $\psi(t)$ is eligible.

Theorem 4.1. $\psi(t)$ is an eligible kernel function.

Proof. Let $N=\frac{M e^{t}}{\left(e^{t}-1\right)^{q+1}}, t>0, q \geq 1$. To prove that the kernel function $\psi(t)$ is eligible, it is sufficient to prove that $\psi(t)$ satisfies (4.2a), (4.2b), (4.2c) and (4.2d).

(i) By using (4.3a) and (4.3b), we have

$$
\begin{aligned}
t \psi^{\prime \prime}(t)+\psi^{\prime}(t) & =t\left[1+\frac{N\left(q e^{t}+1\right)}{e^{t}-1}\right]+t-N \\
& =2 t+\frac{N\left[\left(q e^{t}+1\right) t-e^{t}+1\right]}{e^{t}-1}, t>0, q \geq 1 .
\end{aligned}
$$

Let

$$
f(t)=\left(q e^{t}+1\right) t-e^{t}+1 .
$$

One has

$$
f^{\prime}(t)=(q-1) e^{t}+t q e^{t}+1>0, t>0, q \geq 1,
$$

which implies $f(t)$ is strictly monotonically increasing. Using that $f(t)$ is continuous, it follows that if $t>0$, then $f(t)>f(0)=0$ holds. Hence,

$$
t \psi^{\prime \prime}(t)+\psi^{\prime}(t)>0, t>0, q \geq 1 .
$$

(ii) From (4.3a) and (4.3b), we derive that

$$
\begin{aligned}
t \psi^{\prime \prime}(t)-\psi^{\prime}(t) & =t\left[1+\frac{N\left(q e^{t}+1\right)}{e^{t}-1}\right]-t+N \\
& =\frac{N\left(q e^{t}+1\right) t}{e^{t}-1}+N>0, t>0, q \geq 1 .
\end{aligned}
$$

(iii) Due to (4.3c), we immediately get

$$
\psi^{\prime \prime \prime}(t)=-\frac{M\left[q^{2} e^{3 t}+(3 q+1) e^{2 t}+e^{t}\right]}{\left(e^{t}-1\right)^{q+3}}<0, t>0, q \geq 1 .
$$


(iv) Combining with (4.3a), (4.3b) and (4.3c), it is verified that

$$
\begin{aligned}
& 2 \psi^{\prime \prime}(t)^{2}-\psi^{\prime}(t) \psi^{\prime \prime \prime}(t) \\
& =2\left[1+\frac{N\left(q e^{t}+1\right)}{e^{t}-1}\right]^{2}+(t-N) \frac{N\left[q^{2} e^{2 t}+(3 q+1) e^{t}+1\right]}{\left(e^{t}-1\right)^{2}} \\
& \geq \frac{N^{2}\left[2\left(q e^{t}+1\right)^{2}-\left(q^{2} e^{2 t}+(3 q+1) e^{t}+1\right)\right]}{\left(e^{t}-1\right)^{2}} \\
& =\frac{N^{2}\left[q^{2} e^{2 t}+(q-1) e^{t}+1\right]}{\left(e^{t}-1\right)^{2}}>0, t>0, q \geq 1 .
\end{aligned}
$$

This proves that $\psi(t)$ satisfies (4.2a), (4.2b), (4.2c) and (4.2d), which implies the lemma.

The following Theorem 4.2 gives the influence of the parameter $q$ on the kernel function.

Theorem 4.2. $\psi(t, q)$ increases as parameter $q$ increases for $t \in[0, \infty)$.

Proof. The first derivative of $\psi(t, q)$ with respect to $q$ is as follows

$$
\psi^{\prime}(t, q)=\frac{q(e-1)^{q+1} \ln \frac{e-1}{e^{t}-1}-(e-1)^{q+1}+(e-1)\left(e^{t}-1\right)^{q}}{q^{2} e\left(e^{t}-1\right)^{q}},
$$

where $t>0$ and $q \geq 1$. Consider the function

$$
f(t)=q(e-1)^{q+1} \ln \frac{e-1}{e^{t}-1}-(e-1)^{q+1}+(e-1)\left(e^{t}-1\right)^{q}
$$

and its first derivative

$$
f^{\prime}(t)=-\frac{q e^{t}(e-1)^{q+1}}{e^{t}-1}+q e^{t}(e-1)\left(e^{t}-1\right)^{q-1} .
$$

Obviously, $\psi(1, q)=f(1)=0$. Thus

$$
\begin{aligned}
& f^{\prime}(t)<-\frac{q e^{t}(e-1)^{q+1}}{e-1}+q e^{t}(e-1)(e-1)^{q-1}=0, t \in(0,1), q \geq 1 \\
& f^{\prime}(t) \geq-\frac{q e^{t}(e-1)^{q+1}}{e-1}+q e^{t}(e-1)(e-1)^{q-1}=0, t \in[1, \infty), q \geq 1 .
\end{aligned}
$$

Hence $\psi^{\prime}(t, q)$ is monotonically decreasing with respect to $q$ for $t \in(0,1)$, and monotonically increasing with respect to $q$ for $t \in[1, \infty)$. We also have $\psi^{\prime}(1, q)=0$. Hence, there is $\psi^{\prime}(t, q) \geq 0$ with $t>0$, i.e., $\psi(t, q)$ increases as parameter $q$ increases for $t \in[0, \infty)$. The proof is completed.

The effects of different values of the parameter $q$ on $\psi(t)$ are illustrated in Figure 2.

The following lemmas give several crucial properties which are important in the analysis of the algorithm.

Lemma 4.3. $\frac{1}{2}(t-1)^{2} \leq \psi(t) \leq \frac{1}{2} \psi^{\prime}(t)^{2}$, if $t>0, q \geq 1$.

Proof. By using (2.8) and $\psi^{\prime \prime}(t) \geq 1$, where $q \geq 1$ and $t>0$, we obtain

$$
\psi(t)=\int_{1}^{t} \int_{1}^{\xi} \psi^{\prime \prime}(\zeta) d \zeta d \xi \geq \int_{1}^{t} \int_{1}^{\xi} 1 d \zeta d \xi=\frac{1}{2}(t-1)^{2}
$$




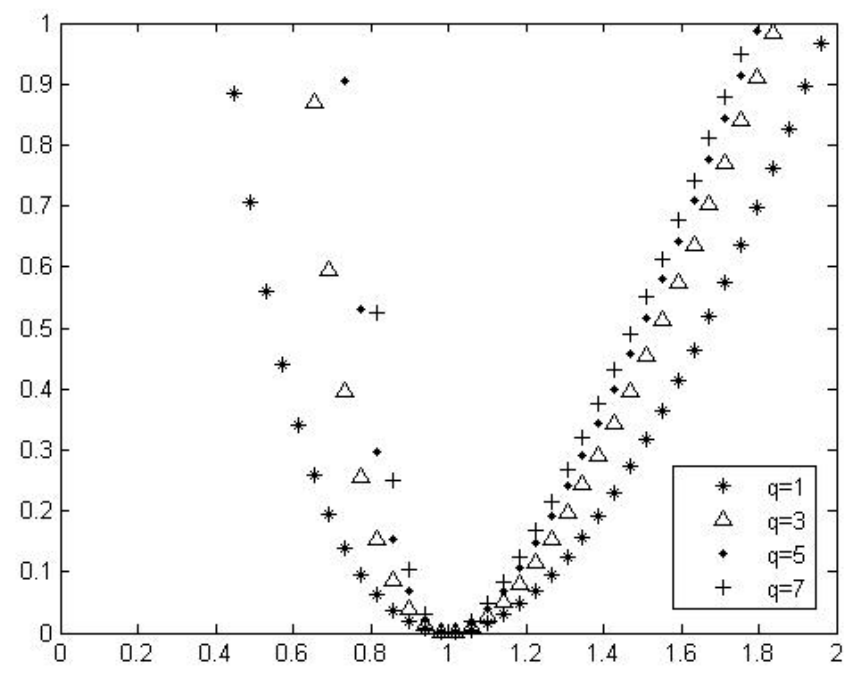

Fig.2 Comparisons of the effects with different $q$ on the kernel function

and

$$
\begin{aligned}
\psi(t) & =\int_{1}^{t} \int_{1}^{\xi} \psi^{\prime \prime}(\zeta) d \zeta d \xi \leq \int_{1}^{t} \int_{1}^{\xi} \psi^{\prime \prime}(\xi) \psi^{\prime \prime}(\zeta) d \zeta d \xi \\
& =\int_{1}^{t} \psi^{\prime \prime}(\xi) \psi^{\prime}(\xi) d \xi=\int_{1}^{t} \psi^{\prime}(\xi) d \psi^{\prime}(\xi)=\frac{1}{2} \psi^{\prime}(t)^{2}
\end{aligned}
$$

This completes the proof.

Lemma 4.4. Suppose that $\rho:[0, \infty) \rightarrow(0,1]$ is the inverse function of the restriction of $-\frac{1}{2} \psi^{\prime}(t)$ to the interval $(0,1]$. Then

$$
\frac{1}{e^{\rho(s)}-1} \leq\left(\frac{2 s+1}{M}\right)^{\frac{1}{q+1}}, q \geq 1 .
$$

Proof. Putting $t=\rho(s)$, which implies $s=-\frac{1}{2} \psi^{\prime}(t)=-\frac{1}{2}\left(t-\frac{M e^{t}}{\left(e^{t}-1\right)^{q+1}}\right)$. Hence

$$
2 s+1 \geq 2 s+t=\frac{M e^{t}}{\left(e^{t}-1\right)^{q+1}} \geq \frac{M}{\left(e^{t}-1\right)^{q+1}}, t \in(0,1], q \geq 1 .
$$

This implies

$$
\frac{1}{e^{\rho(s)}-1}=\frac{1}{e^{t}-1} \leq\left(\frac{2 s+1}{M}\right)^{\frac{1}{q+1}}, t \in(0,1], q \geq 1,
$$

which proves the lemma.

Lemma 4.5. Suppose that $\widetilde{\rho}:[0, \infty) \rightarrow[1, \infty)$ is the inverse function of $\psi(t)$ for $t \in[1, \infty)$. Then

$$
\sqrt{1+2 s} \leq \widetilde{\rho} \leq 1+\sqrt{2 s} .
$$

Proof. Let $t=\widetilde{\rho}$. Then $s=\psi(t)=\frac{t^{2}-1}{2}+\psi_{b}(t), q \geq 1, t \in[1, \infty)$. Since $\psi_{b}(t)$ is monotonically decreasing for $t>1$ and $\psi_{b}(1)=0$, one has

$$
s=\psi(t) \leq \frac{t^{2}-1}{2}, t \in[1, \infty) .
$$


This gives

$$
\sqrt{1+2 s} \leq t=\widetilde{\rho}(s) .
$$

For the right inequality, we use Lemma 4.3 to obtain

$$
s=\psi(t) \geq \frac{1}{2}(t-1)^{2} .
$$

It follows that

$$
\widetilde{\rho}(s)=t \leq 1+\sqrt{2 s} .
$$

The lemma is proved.

Lemma 4.6. $\delta(v) \geq \sqrt{\frac{\Psi(v)}{2}}$.

Proof. By using Lemma 4.3, we obtain

$$
\psi(t) \leq \frac{1}{2} \psi^{\prime}(t)^{2}
$$

which means

$$
\Psi(v)=\sum_{i=1}^{n} \psi\left(v_{i}\right) \leq \frac{1}{2} \sum_{i=1}^{n} \psi^{\prime}\left(v_{i}\right)^{2}=\frac{1}{2}\|\nabla \Psi(v)\|^{2}=2 \delta(v)^{2}
$$

Thus

$$
\delta(v) \geq \sqrt{\frac{\Psi(v)}{2}}
$$

which completes the proof.

\section{COMPLEXITY ANALYSIS OF THE ALGORITHM}

Note that our algorithm include two parts: inner iteration and outer iteration. Before every time the outer iteration of the algorithm begins, that is, before the $\mu$ is updated by the factor $1-\theta, 0<\theta<1$, we have $\Psi(v) \leq \tau$. In the outer iteration, since $\mu$ is updated to $(1-\theta) \mu$, the vector $v=\sqrt{\frac{x s}{\mu}}$ will be divided by the factor $\sqrt{1-\theta}$, thus in general increase the value of $\Psi(v)$. Then the algorithm starts executing the inner iterations if $\Psi(v)>\tau$, and the inner iterations will decrease the value of $\Psi(v)$. The algorithm returns to the outer iteration again when $\Psi(v) \leq \tau$. Repeat the above iteration process until $n \mu \leq \varepsilon$, then we get an $\varepsilon$-solution of (P) and (D).

5.1. Growth behavior. From the above analysis, we conclude that the largest value of $\Psi(v)$ occur after the $\mu$-update, just before the inner iteration begins. What we want is to find an upper bound of $\Psi(v)$ to research the amount of decrease of the barrier function during an inner iteration. Due to the fact that $\psi(t)$ is eligible, and also [13], we have the following lemma.

Lemma 5.1. Let $\widetilde{\rho}$ be defined as in Lemma 4.5, and $v \in R_{++}^{n}, \beta \geq 1$. Then

$$
\Psi(\beta v) \leq n \psi\left(\beta \widetilde{\rho}\left(\frac{\Psi(v)}{n}\right)\right) .
$$


Define

$$
L_{\psi}(n, \theta, \tau):=n \psi\left(\frac{1}{\sqrt{1-\theta}} \widetilde{\rho}\left(\frac{\tau}{n}\right)\right) .
$$

Obviously, if $\Psi(v) \leq \tau$ and $\beta=\frac{1}{\sqrt{1-\theta}}$, then $L_{\psi}(n, \theta, \tau)$ is an upper bound for $\Psi\left(\frac{v}{\sqrt{1-\theta}}\right)$ by using Lemma 5.1 .

Lemma 5.2. Using (5.1), we have

$$
L_{\psi}(n, \theta, \tau) \leq \frac{n \theta+2 \sqrt{2 n \tau}+2 \tau}{2(1-\theta)} .
$$

Proof. Since $\psi_{b}(t)$ is monotonically decreasing for $t \geq 1$, and $\psi_{b}(1)=0$, we get

$$
\psi(t)=\frac{t^{2}-1}{2}+\psi_{b}(t) \leq \frac{t^{2}-1}{2}, t \geq 1, q \geq 1 .
$$

Using the above results, Lemma 4.5 and $\psi(t)$ is monotonically increasing for $t \in[1, \infty)$, we have

$$
\begin{aligned}
L_{\psi}(n, \theta, \tau) & =n \psi\left(\frac{\widetilde{\rho}\left(\frac{\tau}{n}\right)}{\sqrt{1-\theta}}\right) \leq n \psi\left(\frac{1+\sqrt{\frac{2 \tau}{n}}}{\sqrt{1-\theta}}\right) \\
& \leq n \frac{\left(\frac{1+\sqrt{\frac{2 \tau}{n}}}{\sqrt{1-\theta}}\right)^{2}-1}{2}=\frac{n \theta+2 \sqrt{2 n \tau}+2 \tau}{2(1-\theta)} .
\end{aligned}
$$

Hence the lemma is proved.

5.2. Decrease behavior and the choice of step size. This section serves to analyze the decreasing behavior of the barrier function and the choice of step size during an inner iteration. After a damped step, we have

$$
x_{+}=x+\alpha \Delta x=x\left(e+\alpha \frac{\Delta x}{x}\right)=x\left(e+\alpha \frac{d_{x}}{v}\right)=\frac{x}{v}\left(v+\alpha d_{x}\right)
$$

and

$$
s_{+}=s+\alpha \Delta \Delta s=s\left(e+\alpha \frac{\Delta s}{s}\right)=s\left(e+\alpha \frac{d_{s}}{v}\right)=\frac{s}{v}\left(v+\alpha d_{s}\right) .
$$

Thus

$$
v_{+}=\sqrt{\frac{x_{+} s_{+}}{\mu}}=\sqrt{\left(v+\alpha d_{x}\right)\left(v+\alpha d_{s}\right)}
$$

Since $\psi$ is e-convex and Lemma 3.1, one can get

$$
\begin{aligned}
\Psi\left(v_{+}\right) & =\Psi\left(\sqrt{\left(v+\alpha d_{x}\right)\left(v+\alpha d_{s}\right)}\right) \\
& \leq \frac{1}{2}\left(\Psi\left(v+\alpha d_{x}\right)+\Psi\left(v+\alpha d_{s}\right)\right) .
\end{aligned}
$$

Define

$$
f(\alpha):=\Psi\left(v_{+}\right)-\Psi(v),
$$

which denotes the decrease of the barrier function on each inner iteration. An immediate consequence following from (5.3) is

$$
f(\alpha) \leq f_{1}(\alpha):=\frac{1}{2}\left(\Psi\left(v+\alpha d_{x}\right)+\Psi\left(v+\alpha d_{s}\right)\right)-\Psi(v) .
$$


The first second derivatives of $f_{1}(\alpha)$ are given as follows:

$$
\begin{aligned}
& f_{1}^{\prime}(\alpha)=\frac{1}{2} \sum_{i=1}^{n}\left(\psi^{\prime}\left(v_{i}+\alpha d_{x_{i}}\right) d_{x_{i}}+\psi^{\prime}\left(v_{i}+\alpha d_{s_{i}}\right) d_{s_{i}}\right) \\
& f_{1}^{\prime \prime}(\alpha)=\frac{1}{2} \sum_{i=1}^{n}\left(\psi^{\prime \prime}\left(v_{i}+\alpha d_{x_{i}}\right) d_{x_{i}}^{2}+\psi^{\prime \prime}\left(v_{i}+\alpha d_{s_{i}}\right) d_{s_{i}}^{2}\right)
\end{aligned}
$$

We thus have

$$
f(0)=f_{1}(0)=0
$$

and

$$
f_{1}^{\prime}(0)=\frac{1}{2} \nabla \Psi(v)^{T}\left(d_{x}+d_{s}\right)=-\frac{1}{2} \nabla \Psi(v)^{T} \nabla \Psi(v)=-2 \delta(v)^{2} .
$$

With $\delta(v)$ as defined in (4.1), we use the following notations:

$$
v_{1}:=\min (v), \delta:=\delta(v) .
$$

where $v_{1}$ represents the min element-wise of vector. Now we cite several lemmas in [13] which will be used in analyzing the convergence of the algorithm.

Lemma 5.3. ([13] Lemma 4.1) $f_{1}^{\prime \prime}(\alpha) \leq 2 \delta^{2} \psi^{\prime \prime}\left(v_{1}-2 \alpha \delta\right)$.

Lemma 5.4. ([13] Lemma 4.2) If $\alpha$ satisfies the inequality

$$
-\psi^{\prime}\left(v_{1}-2 \alpha \delta\right)+\psi^{\prime}\left(v_{1}\right) \leq 2 \delta
$$

then $f_{1}^{\prime}(\alpha) \leq 0$.

Lemma 5.5. ([13] Lemma 4.3) Using the notions of Lemma 4.4, if step size $\alpha$ satisfies (5.6), then the largest step size $\alpha$ is given by

$$
\bar{\alpha}:=\frac{1}{2 \delta}(\rho(\delta)-\rho(2 \delta)) .
$$

Lemma 5.6. ([13] Lemma 4.4) Let $\rho$ and $\bar{\alpha}$ be defined as in Lemma 5.5. Then

$$
\bar{\alpha} \geq \tilde{\alpha}:=\frac{1}{\psi^{\prime \prime}(\rho(2 \delta))},
$$

and we will use $\tilde{\alpha}$ as the default step size.

Lemma 5.7. If $\tilde{\alpha}$ is defined as in Lemma 5.6, then

$$
\tilde{\alpha} \geq \frac{1}{4(2 q+1)(4 \delta+1)^{\frac{q+2}{q+1}}}, q \geq 1 .
$$

Proof. By using $\rho$ defined in Lemma 4.4, we may assume that $t=\rho(2 \delta)$ for $t \in(0,1]$. Thus we have

$$
\begin{aligned}
\psi^{\prime \prime}(\rho(2 \delta)) & =\psi^{\prime \prime}(t)=1+\frac{M\left(q e^{2 t}+e^{t}\right)}{\left(e^{t}-1\right)^{q+2}} \\
& \leq 1+(4 \delta+1)^{\frac{q+2}{q+1}} M^{-\frac{1}{q+1}}\left(q e^{2 t}+e^{t}\right) \\
& =1+(4 \delta+1)^{\frac{q+2}{q+1}} \frac{e^{\frac{1}{q+1}}}{e-1}\left(q e^{2 t}+e^{t}\right) \\
& \leq 4(2 q+1)(4 \delta+1)^{\frac{q+2}{q+1}}, t \in(0,1], q \geq 1 .
\end{aligned}
$$


Combining the above results and Lemma 5.6, we derive that

$$
\tilde{\alpha}=\frac{1}{\psi^{\prime \prime}(\rho(2 \delta))} \geq \frac{1}{4(2 q+1)(4 \delta+1)^{\frac{q+2}{q+1}}}, q \geq 1 .
$$

This proves the lemma.

Lemma 5.8. ([13] Lemma 4.5) If $\alpha \leq \bar{\alpha}$, then

$$
f(\alpha) \leq-\alpha \delta^{2}
$$

\section{Lemma 5.9.}

$$
f(\tilde{\alpha}) \leq-\frac{\delta^{2}}{\psi^{\prime \prime}(\rho(2 \delta))} \leq-\frac{\delta^{\frac{q}{q+1}}}{4(2 q+1)\left(4+\frac{1}{\delta}\right)^{\frac{q+2}{q+1}}}, q \geq 1 .
$$

Proof. From Lemma 5.6, 5.7 and 5.8, we have

$$
\begin{aligned}
f(\tilde{\alpha}) & \leq-\frac{\delta^{2}}{\psi^{\prime \prime}(\rho(2 \delta))} \leq-\frac{\delta^{2}}{4(2 q+1)(4 \delta+1)^{\frac{q+2}{q+1}}} \\
& \leq-\frac{\delta^{\frac{q}{q+1}}}{4(2 q+1)\left(4+\frac{1}{\delta}\right)^{\frac{q+2}{q+1}}}, q \geq 1 .
\end{aligned}
$$

Hence, the results of this lemma hold.

5.3. Iteration complexity. Our goal in this section is to analyze the convergence of the method. The question is to count the number of iterations are required to return to the situation where $\Psi(v) \leq \tau$. To investigate this, we define the value of $\Psi(v)$ after each $\mu$-update as $\Psi_{0}$, and the subsequent values during inner iterations as $\Psi_{\kappa}, \kappa=1,2, \ldots, K$. Thus $K$ is the number of iterations in the inner iteration after once $\mu$-update.

By using Lemma 5.1, Lemma 5.2 and the definition of $\Psi_{0}$, we have $L \Psi \geq \Psi_{0} \geq \Psi \geq \tau$. In what follows, we assume $L_{\psi} \geq \Psi_{0} \geq \Psi \geq \tau \geq 2$. From Lemma 4.6, we deduce that $\delta(v) \geq \sqrt{\frac{\Psi}{2}} \geq 1$. Substitution into (5.10) gives

$$
f(\tilde{\alpha}) \leq-\frac{\delta^{\frac{q}{q+1}}}{4(2 q+1)\left(4+\frac{1}{\delta}\right)^{\frac{q+2}{q+1}}} \leq-\frac{\Psi^{\frac{q}{2(q+1)}}}{2^{\frac{1}{2}} \times 5^{\frac{3}{2}} \times 4(2 q+1)} \leq-\frac{\Psi^{\frac{q}{2(q+1)}}}{64(2 q+1)}, q \geq 1,
$$

which implies

$$
\Psi_{\kappa+1} \leq \Psi_{\kappa}-\frac{\Psi_{\kappa}^{\frac{q}{2(q+1)}}}{64(2 q+1)}, \kappa=0,1,2, \ldots, K-1 .
$$

To derive an upper bound for the total number of inner iterations after an outer iteration, we give the following technical lemma. We refer the reader its elementary proof to [11].

Lemma 5.10. Suppose that $t_{0}, t_{1}, \ldots, t_{K}$ is a sequence of positive numbers such that

$$
t_{\kappa+1} \leq t_{\kappa}-\beta t_{\kappa}^{1-\gamma}, \kappa=0,1,2, \ldots, K-1,
$$

where $\beta>0$ and $0<\gamma \leq 1$. Then $K \leq\left\lfloor\frac{t_{0}^{\gamma}}{\beta \gamma}\right\rfloor$. 
Lemma 5.11. The following inequality holds:

$$
K \leq 128(2 q+1) \Psi_{0}^{\frac{q+2}{2(q+1)}}, q \geq 1 .
$$

Proof. Let $t_{\kappa}=\Psi_{\kappa}, \beta=\frac{1}{64(2 q+1)}, \gamma=\frac{q+2}{2(q+1)}$. Using Lemma 5.10 and substitution gives, we have

$$
\begin{aligned}
K \leq \frac{\Psi_{0}^{\gamma}}{\beta \gamma} & =\frac{128(2 q+1)(q+1) \Psi_{0}^{\frac{q+2}{2(q+1)}}}{q+2} \\
& \leq 128(2 q+1) \Psi_{0}^{\frac{q+2}{2(q+1)}}, q \geq 1 .
\end{aligned}
$$

This implies the lemma.

From [2], the total number of iterations of the algorithm is given below:

$$
K \cdot \frac{1}{\theta} \log \frac{n}{\varepsilon}
$$

where $\frac{1}{\theta} \log \frac{n}{\varepsilon}$ is the number of barrier parameter updates. Recall that $L_{\psi} \geq \Psi_{0}$, and $L_{\psi}$ is bounded by Lemma 5.2. Combining the above results and Lemma 5.11, we immediately obtain the following theorem.

Theorem 5.12. The total number of iterations required by the algorithm is at most

$$
128 \frac{(n \theta+2 \sqrt{2 n \tau}+2 \tau)^{\frac{q+2}{2(q+1)}}(2 q+1)}{\theta[2(1-\theta)]^{\frac{q+2}{2(q+1)}}} \log \frac{n}{\varepsilon}, q \geq 1 .
$$

Setting $\tau=O(n)$ and $\theta=\Theta(1)$, as a consequence, we conclude that the iteration bound of the largeupdate method is

$$
O\left(q n^{\frac{q+2}{2(q+1)}} \log \frac{n}{\varepsilon}\right) .
$$

Note that $\psi(t)$ is precisely the kernel function proposed by Bai, Roos and Ghami [24] if $q=1$, and the iteration bound for large-update method is $O\left(n^{\frac{3}{4}} \log \frac{n}{\varepsilon}\right)$. By choosing $q=O(\log n)$, the iteration bound becomes $O\left(\sqrt{n} \log n \log \frac{n}{\varepsilon}\right)$, which is the best known iteration bound for such methods.

\section{NUMERICAL RESULTS}

In this section, some numerical results of the large-update IPMs for LO are given. Several problems of LO are considered as below:

Problem 6.1. [32]

$$
A=\left(\begin{array}{ccccccc}
-8 & -2 & -8 & 6 & -3 & -1 & 7 \\
-5 & 10 & -2 & -9 & 4 & -4 & -5 \\
-8 & 1 & -1 & -3 & -8 & -6 & -6 \\
9 & 2 & 7 & 1 & 5 & -4 & -7 \\
-4 & -3 & -4 & -2 & 6 & -3 & -1
\end{array}\right), x^{0}=\left(\begin{array}{l}
9 \\
9 \\
1 \\
5 \\
5 \\
2 \\
1
\end{array}\right), s^{0}=\left(\begin{array}{l}
1 \\
5 \\
8 \\
2 \\
9 \\
9 \\
6
\end{array}\right)
$$


Problem 6.2. By using MATLAB2012b, we generate a random row full rank matrix $A$, where the dimension of matrix A is $50 \times 60, x^{0}=(1,1, \cdots, 1)^{T}$ and $s^{0}=(1,1, \cdots, 1)^{T}$. Thus we obtain a corresponding test problem.

Problem 6.3. By using MATLAB2012b, we generate a random row full rank matrix $A$, where the dimension of matrix A is $100 \times 120, x^{0}=(1,1, \cdots, 1)^{T}$ and $s^{0}=(1,1, \cdots, 1)^{T}$. Thus we obtain a corresponding test problem.

In the implementation, we set threshold parameter $\tau=n$, accuracy parameter $\varepsilon=10^{-6}$. The numerical results are obtained by MATLAB 2012b.

The best iteration numbers for Problems 6.1, 6.2 and 6.3 based on our kernel function with different values of the parameters $q$ and $\theta$ are stated in Table 2-4, where $q \in\{1,2,3,5,10\}$ and $\theta \in$ $\{0.2,0.4,0.6,0.8,0.99\}$.

Table 2 Numerical results based on our kernel function for problem 6.1

\begin{tabular}{|c|ccccc|c|}
\hline \multirow{2}{*}{$\mathrm{q}$} & $\theta$ & & & & \multirow{2}{*}{ Average } \\
\cline { 2 - 5 } & 0.2 & 0.4 & 0.6 & 0.8 & 0.99 & \\
\hline 1 & 94 & 45 & 29 & 19 & 11 & 39.6 \\
1.5 & 93 & 45 & 29 & 19 & 11 & 39.4 \\
2 & 94 & 46 & 29 & 20 & 11 & 40 \\
2.5 & 95 & 47 & 30 & 21 & 15 & 41.6 \\
3 & 96 & 47 & 30 & 22 & 15 & 42 \\
\hline
\end{tabular}

Table 3 Numerical results based on our kernel function for problem 6.2

\begin{tabular}{|c|ccccc|c|}
\hline \multirow{2}{*}{$\mathrm{q}$} & \multicolumn{1}{|c|}{$\theta$} & & & & \multirow{2}{*}{ Average } \\
\cline { 2 - 5 } & 0.2 & 0.4 & 0.6 & 0.8 & 0.99 & \\
\hline 1 & 90 & 45 & 28 & 20 & 11 & 38.8 \\
1.5 & 89 & 44 & 28 & 20 & 11 & 38.4 \\
2 & 90 & 45 & 28 & 21 & 12 & 39.2 \\
2.5 & 90 & 45 & 29 & 21 & 12 & 39.4 \\
3 & 91 & 46 & 30 & 22 & 13 & 40.4 \\
\hline
\end{tabular}

Furthermore, we give a series of numerical results in Table 5 to compare the practical computational efficiency of the methods based on different kernel functions and $\theta$ for Problem 6.1, Problem 6.2, and 
Table 4 Numerical results based on our kernel function for problem 6.3

\begin{tabular}{|c|c|c|c|c|c|c|}
\hline \multirow{2}{*}{$\mathrm{q}$} & \multicolumn{5}{|l|}{$\theta$} & \multirow{2}{*}{ Average } \\
\hline & 0.2 & 0.4 & 0.6 & 0.8 & 0.99 & \\
\hline 1 & 94 & 47 & 30 & 21 & 14 & 41.2 \\
\hline 1.5 & 93 & 46 & 30 & 21 & 15 & 41 \\
\hline 2 & 94 & 46 & 30 & 21 & 16 & 41.4 \\
\hline 2.5 & 94 & 47 & 31 & 22 & 17 & 42.2 \\
\hline 3 & 95 & 48 & 32 & 23 & 18 & 43.2 \\
\hline
\end{tabular}

Problem 6.3. All numerical results in Table 5 include iteration numbers (iter), the duality gap (Gap) and the CPU time (CPU). Some kernel functions we used in the experiments are as follows:

$$
\begin{aligned}
& \psi_{\text {new }}(t)=\frac{t^{2}-1}{2}+\frac{(e-1)^{q+1}}{q e\left(e^{t}-1\right)^{q}}-\frac{e-1}{q e}, q \geq 1, \\
& \psi_{1}(t)=\frac{t^{2}-1}{2}-\log t, \psi_{2}(t)=\frac{t^{2}-1}{2}+\frac{t^{-1}-t}{2}, \\
& \psi_{3}(t)=\frac{t^{2}-1}{2}+\frac{6}{\pi} \tan (h(t)), h(t)=\frac{\pi(1-t)}{4 t+2}, \\
& \psi_{4}(t)=\frac{t^{2}-1}{2}-\int_{1}^{t}\left(\frac{e-1}{e^{x}-1}\right)^{p} d x, p \geq 1, \\
& \psi_{5}(t)=\frac{t^{2}-1}{2}+\frac{1}{q^{2}}\left(\frac{q}{t}-1\right) e^{q\left(\frac{1}{t}-1\right)}-\frac{q-1}{q^{2}}, q \geq 1 .
\end{aligned}
$$

The first kernel function $\psi_{\text {new }}(t)$ is proposed in this paper, and it is exactly the kernel function presented by Bai, Roos and Ghami [24] for $q=1 . \psi_{1}(t)$ is the classical logarithmic kernel function. $\psi_{2}(t)$ is a self-regular kernel function presented by Peng, Roos and Terlaky [33]. $\psi_{3}(t)$ is a trigonometric kernel function [14]. $\psi_{4}(t)$ [34] and $\psi_{5}(t)$ [29] are parameterized kernel functions, which obtain the best iteration complexity for $q=O(\log n)$.

From the numerical results of Tables 2-5, we can draw the following conclusions:

- The results in Table 2-4 show that the larger $\theta$ is, the better iteration numbers will be.

- From the Table 2-4, the best iteration results of methods for Problems 6.1-6.3 are obtained at $q=1.5$. Compared with the original kernel function, that is, when the parameter $q=1$, the numerical result is improved.

- Note that the number of iteration results using $\psi_{4}(t)$ is efficient, but it consumes significantly more CPU time than other kernel functions, probably because its integral form leads to more complex operations.

- From Table 2-5, our kernel function has better numerical results than the other kernel functions.

These results imply that our kernel function is quite efficient and promising. 
Table 5 Numerical results based on different kernel functions

\begin{tabular}{|c|c|c|c|c|c|c|c|c|c|c|c|c|}
\hline \multirow{2}{*}{\multicolumn{2}{|c|}{$\psi_{i}(\theta=0.99)$}} & \multicolumn{3}{|c|}{ Problem 6.1} & \multicolumn{3}{|c|}{ Problem 6.2} & \multicolumn{3}{|c|}{ Problem 6.3} & \multicolumn{2}{|c|}{ Average } \\
\hline & & Iter & Gap & CPU & Iter & Gap & CPU & Iter & Gap & CPU & Iter & CPU \\
\hline$\psi_{1}$ & & 13 & $5.0 \mathrm{e}-08$ & 0.0126 & 12 & $1.4 \mathrm{e}-08$ & .0138 & 15 & $1.2 \mathrm{e}-08$ & 0297 & 3.3 & .0187 \\
\hline$\psi_{2}$ & & 15 & $6.0 \mathrm{e}-08$ & 0.0133 & 12 & $1.4 \mathrm{e}$ & 0157 & 16 & $1.2 \mathrm{e}-08$ & 0.0332 & 4.3 & 0207 \\
\hline$\psi_{3}$ & & 15 & $6.0 \mathrm{e}-08$ & 0.0152 & 13 & $1.4 \mathrm{e}-08$ & 0.0222 & 16 & $1.2 \mathrm{e}-08$ & 0.0393 & 14.7 & 0.0256 \\
\hline \multirow{5}{*}{$\psi$} & $p-1$ & 11 & $6.0 \mathrm{e}-08$ & 1.5542 & 12 & $1.4 \mathrm{e}-08$ & 1.9576 & 14 & $1.2 \mathrm{e}-08$ & 3.7576 & 12.3 & 2.4231 \\
\hline & $\mathrm{p}=1.5$ & 11 & $6.0 \mathrm{e}-08$ & 1.6322 & 11 & $1.4 \mathrm{e}-08$ & 2.1055 & 14 & $1.2 \mathrm{e}-08$ & 3.9243 & 12 & 2.5540 \\
\hline & $\mathrm{p}=2$ & 11 & $6.0 \mathrm{e}-08$ & 1.7125 & 11 & $1.4 \mathrm{e}-08$ & 2.4344 & 15 & $1.2 \mathrm{e}-08$ & 4.5125 & 12.3 & 2.8865 \\
\hline & & 11 & & 52 & 11 & 1.4 & 11 & 15 & & & 12.3 & 132 \\
\hline & & 11 & & & 13 & & & 17 & & & 13.7 & 44 \\
\hline \multirow{5}{*}{$\psi_{5}$} & $\mathrm{q}=1$ & 14 & $6.0 \mathrm{e}-08$ & 0.0105 & 12 & $1.4 \mathrm{e}-08$ & 0.0123 & 16 & $1.2 \mathrm{e}-08$ & 0.0209 & 14 & 0.0146 \\
\hline & $q=1.5$ & 14 & $6.0 \mathrm{e}-08$ & 0.0111 & 12 & $1.4 \mathrm{e}-08$ & 0.0122 & 17 & $1.2 \mathrm{e}-08$ & 0.0220 & 14.3 & 0.0151 \\
\hline & $q=2$ & 15 & $6.0 \mathrm{e}-08$ & 0.0100 & 13 & $1.4 \mathrm{e}-08$ & 0.0112 & 17 & $1.2 \mathrm{e}-08$ & 0.0232 & 15 & 0.0138 \\
\hline & $q=2.5$ & 15 & $6.0 \mathrm{e}-08$ & 0.0124 & 13 & $1.4 \mathrm{e}-08$ & & 18 & $1.2 \mathrm{e}-08$ & 0.0226 & 15.3 & 0.0173 \\
\hline & $q=3$ & 15 & $6.0 \mathrm{e}-08$ & 0.0131 & 13 & $1.4 \mathrm{e}$ & & 18 & $1.2 \mathrm{e}-08$ & 0.0300 & 15.3 & 0.0190 \\
\hline \multirow{5}{*}{$\psi_{\text {new }}$} & & 11 & $6.0 \mathrm{e}-08$ & 0.0105 & 11 & $1.4 \mathrm{e}-08$ & 0.0128 & 14 & $1.2 \mathrm{e}-08$ & 0.0203 & 12 & 0.0145 \\
\hline & $\mathrm{q}=1.5$ & 11 & $6.0 \mathrm{e}-08$ & 0.0102 & 11 & $1.4 \mathrm{e}-08$ & 0.0125 & 15 & $1.2 \mathrm{e}-08$ & 0.0197 & 12.3 & 0.0141 \\
\hline & $q=2$ & 11 & $6.0 \mathrm{e}-08$ & 0.0112 & 12 & $1.4 \mathrm{e}-08$ & 0.0131 & 16 & $1.2 \mathrm{e}-08$ & 0.0212 & 13 & 0.0152 \\
\hline & $q=2.5$ & 15 & $6.0 \mathrm{e}-08$ & 0.0115 & 12 & $1.4 \mathrm{e}-08$ & 0.0142 & 17 & $1.2 \mathrm{e}-08$ & 0.0233 & 14.7 & 0.0163 \\
\hline & $q=3$ & 15 & $6.0 \mathrm{e}-08$ & 0.0129 & 13 & $1.4 \mathrm{e}-08$ & 0.0145 & 18 & $1.2 \mathrm{e}-08$ & 0.0254 & 15.3 & 0.0176 \\
\hline
\end{tabular}

\section{CONCLUDING REMARKS}

In this paper, we constructed a new parameterized kernel function, which is a kind of generalization of the one used in [24]. We use it to design a primal-dual IPM for LO, and the properties of the kernel function have been analyzed. As a result, the iteration bound in [24] is improved from $O\left(n^{\frac{3}{4}} \log \frac{n}{\varepsilon}\right)$ to $O\left(\sqrt{n} \log n \log \frac{n}{\varepsilon}\right)$ for the large-update method. We also presented numerical results, and the practical performance seems quite promising and significant based on our kernel function.

\section{Funding}

This paper was supported by Natural Science Foundation of China (Grant No.71471102).

\section{REFERENCES}

[1] N.K. Karmarkar, A New Polynomial-Time Algorithm for Linear Programming, Combinatorica 4 (1984), 373-395.

[2] C. Roos, T. Terlaky, J.-Ph. Vial, Theory and Algorithms for Linear Optimization. An Interior-Point Approach. John Wiley \& Sons, Chichester, 1997.

[3] S.J. Wright, Primal-Dual Interior-Point Methods, SIAM, Philadelphia, 1997.

[4] Y. Ye, Interior Point Algorithms, Theory and Analysis, John Wiley \& Sons, Chichester, UK, 1997.

[5] M. Kojima, S. Mizuno, A. Yoshise, A Primal-Dual Interior Point Algorithm for Linear Programming. Interior Point and Related Methods. Springer, New York, 1989.

[6] E.D. Andersen, J. Gondzio, Cs. Mészáros, X. Xu, Implementation of Interior-Point Methods for Large Scale Linear Programs, in Interior Point Methods of Mathematical Programming, pp. 189-252, Springer, US, 1996.

[7] N. Megiddo, Progress in Mathematical Programming Bzteriar Point and Related Methods, pp. 131-158, Springer, New York, 1989. 
[8] C.C. Gonzaga, Path following methods for linear programming, SIAM Rev. 34 (1992), 167-227.

[9] C. Roos, J.-Ph. Vial, A polynomial method of approximate centers for linear programming, Math. Program. 54 (1992), 295-305.

[10] Y. Nesterov, A. Nemirovskii, Interior-Point Ploynomial Methods in Convex Programming, SIAM, Philadelphia, 1994.

[11] J.M. Peng, C. Roos, T. Terlaky, Self-regular functions and new search directions for linear and semidefinite optimization, Math. Program. 93 (2001), 129-171.

[12] J.M. Peng, C. Roos, T. Terlaky, A new and efficient large-update interior-point method for linear optimization, Vychisl. Tekhnol. 6 (2001), 61-80.

[13] Y.Q. Bai, M.E. Ghami, C. Roos, A comparative study of kernel functions for primal-dual interior-point algorithms in linear optimization, SIAM J. Optim. 15 (2004), 101-128.

[14] M.E. Ghami, Z.A. Guennounb, S. Bouali, T. Steihaug, Interior-point methods for linear optimization based on a kernel function with a trigonometric barrier term, J. Comput. Appl. Math. 236 (2012), 3613-3623.

[15] X. Li, M.W. Zhang, Interior-point algorithm for linear optimization based on a new trigonometric kernel function, Oper. Res. Lett. 43 (2015), 471-475.

[16] P. Ji, M.W. Zhang, X. Li, A primal-dual large-update interior-point algorithm for $P *(\kappa)$-LCP based on a new class of kernel functions, Acta Math. Appl. Sin., Engl. Ser. 34 (2018), 119-134.

[17] S. Fathihafshejani, A.F. Jahromi, M.R. Peyghami, A unified complexity analysis of interior point methods for semidefinite problems based on trigonometric kernel functions, Optimization, 67 (2018), 113-137.

[18] S.F. Hafshejani, M. Fatemi, M.R. Peyghami, An interior-point method for $P_{*}(\kappa)$-linear complementarity problem based on a trigonometric kernel function, J. Comput. Appl. Math. 48 (2015), 111-128.

[19] Y.Q. Bai, G.Q. Wang, Primal-dual interior-point algorithms for second-order cone optimization based on a new parametric kernel function, Acta Math. Sin. Engl. Ser. 23 (2007), 2027-2042.

[20] M.W. Zhang, A large-update interior-point algorithm for convex quadratic semi-definite optimization based on a new kernel function. Acta Math. Sin. Engl. Ser. 28 (2012), 2313-232.

[21] B. Kheirfam, Primal-dual interior-point algorithm for semidefinite optimization based on a new kernel function with trigonometric barrier term, Numer. Algo. 61 (2012), 659-680.

[22] Y.Q. Bai, G.Q. Wang, C. Roos, Primal-dual interior-point algorithms for second-order cone optimization based on kernel functions, Nonlinear Anal. 70 (2009), 3584-3602.

[23] Y.Q. Bai, M.E. Ghami, C. Roos, A new efficient large-update primal-dual interior-point method based on a finite barrier, SIAM J. Optim. 13 (2003), 766-782.

[24] Y.Q. Bai, C. Roos, M.E. Ghami, A primal-dual interior-point method for linear optimization based on a new proximity function, Optim. method softw. 17 (2002), 985-1008.

[25] J.M. Peng, C. Roos, T. Terlaky, Self-Regularity: A New Paradigm for Primal-Dual Interior-Point Algorithms, Princeton University Press, Princeton, 2002.

[26] G.M. Cho, Y.Y. Cho, Y.H. Lee, A primal-dual interior-point algorithm based on a new kernel function, Anziam J. 51 (2001), 476-491.

[27] M. Achache, A new parameterized kernel function for LO yielding the best known iteration bound for a large-update interior-point algorithm, Afrika Matematika, 27 (2016), 591-601.

[28] Y.Q. Bai, J.L. Guo, C. Roos, A new kernel function yielding the best known interation bounds for primal-dual interiorpoint algorithms, Acta Math. Sin. Engl. Ser. 25 (2009), 2169-2178.

[29] Y.Q. Bai, W. Xie, J. Zhang, New parameterized kernel functions for linear optimization. J. Global Optim. 54 (2012), 353-366.

[30] G. Sonnevend, An "analytical center" for polyhedrons and new classes of global algorithms for linear (smooth, convex) programming, Lect. Notes Control Inf. Sci. 84 (1986), 866-875.

[31] J. Renegar, A Mathematical View of Interior-Point Methods in Convex Optimization, SIAM, Philadelphia, 2001.

[32] D. Darvay, P.R. Takács, New method for determining search directions for interior-point algorithms in linear optimization, Optim. Lett., 12 (2018), 1099-1116.

[33] J.M. Peng, C. Roos, T. Terlaky, New complexity analysis of the primal-dual newton method for linear optimization, Ann. Oper. Res. 99 (2000), 23-39. 
[34] S. Fathi-Hafshejani, A. Fakharzadeh, An interior-point algorithm for semidefinite optimization based on a new parametric kernel function, J. Nonlinear Funct. Anal. 2018 (2018), Article ID 14. 